\title{
Los cuentos novela en Aragón y La Rioja (España)*
}

\author{
Carlos González Sanz \\ Archivo Pirenaico de Patrimonio Oral \\ bozalongo@hotmail.com
}

\begin{abstract}
RESUMEN
Como paso previo para la presentación del repertorio de los relatos recogidos y de los cuentos tipo catalogados en Aragón y La Rioja —en este último caso, a partir de la colaboración del autor en la clasificación de los cuentos recopilados y editados por el folclorista Javier Asensio en esta comunidad autónoma-, se propone una reflexión acerca del subgénero de los cuentos novela. Para ello se parte de la definición que Carme Oriol ofrece en su obra Introducció a l'etnopoètica. Teoria i formes del folklore en la cultura catalana y se tienen en cuenta las críticas a la etnopoética que Luis Beltrán expone en «Géneros y estéticas en la literatura tradicional», de las que se concluye que, junto a las características de los cuentos novela o cuentos de ingenio que señala Carme Oriol, deben considerarse también su carácter de género bajo, su naturaleza mixta serio-cómica y la naturaleza idílica de los relatos del conflicto familiar. Por lo que respecta a los cuentos novela aragoneses y riojanos, se destaca, en particular, que una amplia mayoría de ellos responden claramente a la función educativa y sapiencial propia de este género, siendo el ingenio y el buen consejo los principales valores que ofrecían a sus receptores.
\end{abstract}

\section{PALABRAS CLAVE}

cuentos folclóricos; cuentos novela; etnopoética; Aragón; La Rioja

\footnotetext{
* Este texto fue presentado en el encuentro del Groupe de Recherche Européen sur les Narrations Orales, celebrado en Atenas en el marco del congreso Classifying Folktales. The Romantic Folktales-Novellae. Boundaries and Classification, organizado por el Centre for the Study and Dissemination of Myths and Folktales entre el 8 y el io de mayo de 2008. Dado que había permanecido inédito hasta la fecha, he decido publicarlo en homenaje y recuerdo al añorado maestro y amigo Josep M. Pujol, a quien en gran medida debo mis conocimientos en materia de folclore y, en particular, del cuento folclórico. Puesto que se trataba de un texto para ser sometido a debate, he decidido también no modificar su contenido; esto es, se presenta tal cual fue leído en el citado encuentro, únicamente con algunas ligeras correcciones de estilo.
} 


\title{
RESUM
}

Com a pas previ per a presentar el repertori dels relats recollits i dels contes-tipus catalogats a Aragó i La Rioja - en aquest darrer cas, a partir de la col-laboració de l'autor en la classificació de les rondalles recopilades i editades pel folklorista Javier Asensio en aquesta comunitat autònoma-, es proposa una reflexió sobre el subgènere de les rondalles d'enginy. Es parteix per a fer-ho de la definició que Carme Oriol dóna en la seva obra Introducció a l'etnopoètica. Teoria i formes del folklore en la cultura catalana $i$ es tenen en compte les crítiques a l'etnopoètica que Luis Beltrán exposa a «Géneros y estéticas en la literatura tradicional», concloent que, juntament amb les característiques de les rondalles d'enginy que assenyala Carme Oriol, s'han de considerar també el seu caràcter de gènere baix, la seva naturalesa mixta seriosa i còmica i la naturalesa idíl.lica dels relats del conflicte familiar. Pel que fa a les rondalles d'enginy aragoneses i de La Rioja, es destaca, en particular, que una àmplia majoria d'aquestes responen clarament a la funció educativa i sapiencial, pròpia d'aquest gènere, sent l'enginy i el bon consell els principals valors que aquestes rondalles oferien als seus receptors.

\section{PARAules Clau}

rondalles; rondalles d'enginy; etnopoètica; Aragó; La Rioja

\begin{abstract}
This article prepares the ground for the presentation of the repertoire of short stories and types collected and classified in Aragón and La Rioja, a process in which the author assisted by classifying the tales compiled and edited by the folklorist Javier Asensio in those regions. In doing so, the present article reflects on the subgenre of the realistic tale (novelle), using as its starting point the definition given by Carme Oriol in her work Introducció a l'etnopoètica. Teoria i formes del folklore en la cultura catalana and Luis Beltrán's critique of ethnopoetry in his Géneros y estéticas en la literatura tradicional. The article concludes that, in addition to possessing the characteristics of novelles or tales of wit mentioned by Carme Oriol, the Aragonese and Riojan tales are also characterised by the low status of the genre, their mixed serious-comic nature and their idyllic depictions of family conflict. Of particular note among the Aragonese and Riojan novelles is that a great number of them clearly have the educational and intellectual purpose that is typical of the genre, with sharp-wittedness and good advice being the main values that these tales offer to their readers.
\end{abstract}

\section{KEYWORDS}

Folktales; Realistic Tales (Novelle); Etnopoetry; Aragon; La Rioja

REBUT: 24.IO.2OI4 | ACCEPTAT: I4.II.2OI4 


\section{Introducción: el problema de la definición de los cuentos novela}

En nuestra primera reunión en Toulouse, en noviembre de 2003, Hans-Jörg Uther nos ofreció lo que suponía un adelanto de su revisión del Catálogo Internacional del Cuento Tipo. Refiriéndose entonces a las principales críticas que había recibido la clasificación de Aarne-Thompson, señalaba entre otras que «la définition des genres et la classification en fonction de personnages ne sont constantes ni thématiquement ni structurellement. Par exemple AaTh 850-999 (Contes nouvelles) ne renvoie à aucun genre distinct» (Uther 2005: 225).

Resulta revelador que, al aludir al problema de la definición de los géneros del cuento folclórico dentro de la clasificación de Aarne y Thompson, el ejemplo aparentemente más adecuado, el primero en el que ha pensado Hans-Jörg Uther, haya sido, precisamente, el del subgénero que nos ocupa en este nuevo encuentro o reencuentro en el magnífico marco de Atenas. Merece la pena, por tanto, detenerse, antes de entrar en el objeto de nuestra exposición, en la definición de la categoría de los cuentos novela para valorar en qué medida tal categoría es o no un subgénero del cuento folclórico y qué consecuencias se derivan de ello en relación con la propia clasificación del cuento tipo.

Por supuesto, si consideramos — como es mi caso- que la clasificación internacional tiene o debe tener como principal propósito ordenar un corpus de textos con fines prácticos, no hay en realidad ningún problema que considerar, o bien este apenas tiene importancia. Serían cuentos novela los tipos que todos conocemos como tales y, por más arbitraria que pueda resultarnos la ordenación, esta no pierde por ello la utilidad para la que ha sido creada. Sin embargo, el problema, como señala Luis Beltrán en un artículo que interesa enormemente a los presentes -y cuya lectura, por tanto, me permito recomendar (Beltrán 2002) —, , es que la lista creada originalmente por Antti Aarne fue elevada a un estatus muy superior cuando Thompson, en su revisión, decidió denominarla clasificación. Como señala Luis Beltrán, el giro no es de poca importancia y afecta de manera muy seria a nuestra propia profesión, pues a la vista de una clasificación no podemos conformarnos con ser capaces de localizar un dato, sino que es preciso saber qué relaciones se dan entre ese y otros datos y qué tipo de almacenamiento o de clasificación de elementos humanos se debe mantener en una determinada situación para alcanzar un fin preciso (Beltrán 2002: 68).

Cabe, por tanto, abordar el problema — para dejarlo sobre la mesa de trabajode la definición de los cuentos novela y, sobre todo, de su estatus como subgénero del cuento folclórico. Al respecto, interesa plantearse también, como hace Luis Beltrán en el artículo citado, si los géneros del folclore son simplemente categorías mecánicas o estructurales o si, por el contrario, se corresponden con fenómenos de la vida social y cultural. Creo que todos los presentes estaremos de acuerdo en lo último, pues conocemos el carácter utilitario del folclore, lo que justifica que esta disciplina sea la única en la que la categoría de género no se ha desprestigiado ni se considera innecesaria, dado que, al contrario de lo que ocurre en la literatura, sigue siendo útil, precisamente, para la propia clasificación (Beltrán 2002: 70).

I. El artículo en cuestión tiene como objeto criticar la clasificación de la etnopoética de Heda Jason, a la que, si bien halaga, reconociendo la necesidad de este tipo de propuestas, achaca ciertos problemas, que, como se verá, afectan en particular a la definición del modo realista, en el que precisamente se encuadran los cuentos novela. 
No obstante, volviendo a las palabras de Uther, cabe preguntarse si todos los subgéneros establecidos por Aarne-Thompson se corresponden realmente con fenómenos de la vida social o si alguno de ellos — quizás sea el caso de los cuentos novela - es una pura categoría mecánica, creada para llenar un vacío en una construcción teórica. En relación con ello, debemos afrontar el problema estudiándolo en términos diacrónicos o, si se prefiere, históricos; es preciso recordar, como hace Luis Beltrán, que la irrupción de la historia en el mundo de las tradiciones y la más reciente llegada de la modernidad — con su entorno de pensamiento individualista - han supuesto un doble proceso de desintegración de los géneros tradicionales, que, si bien ha permitido a algunos, como el cuento folclórico, su supervivencia en el campo literario histórico, ha hecho que, dentro de la esfera de la cultura elevada, se diluyan sus raíces sociales y se hagan menos reconocibles sus vínculos con la vida. A este respecto, resulta sugerente recordar que el filólogo clásico alemán Erwin Rohde consideró que del primer proceso de desintegración al que nos hemos referido, que afectó a los géneros tradicionales del mundo griego, nacieron las bases de la creación de la novela; en relación con el segundo de los procesos de desintegración, el producido por la llegada de la modernidad, Bajtin consideró que se había producido una «novelización» de los géneros (Beltrán 2002: 70-7I).

Llegados a este punto, merece la pena referirse a un problema que podría ser un síntoma en relación con la dificultad para encontrar la raíz social de los cuentos novela en la actualidad. Me refiero a su propia denominación, que, al menos en español, resulta bastante problemática. Se dirá que este es un asunto de poca importancia, que solo atañe a nuestra cultura y que algunos considerarán simple muestra de la mala calidad o la escasez de traducciones en España; sin embargo, desde mi punto de vista, se produce también por una incomprensión del presunto género que aquí nos ocupa y por una problemática relación de este con el término novela. En efecto, la denominación novelle (romantic tales) del texto inglés de Aarne-Thompson ha sido traducida frecuentemente en español de manera literal - podríamos hablar aquí de un caso de falso amigo-, tanto por lo que respecta al término novela como por la expresión cuentos románticos. ${ }^{2}$ En ambos casos se puede apreciar un error de bulto, que confunde lo novelesco con lo romántico y que se ha tratado de subsanar con híbridos como cuentos novelescos —utilizado por el estadounidense Aurelio M. Espinosa (Espinosa I996-I997) - o con la denominación consagrada por el catálogo de Julio Camarena y Maxime Chevalier (Camarena-Chevalier 2003) de cuentos novela, quizá la más acertada de todas.

No obstante, como decía al principio, todos estos términos muestran no solo cierta incapacidad para la traducción del término inglés, sino una asimilación un tanto forzada de una categoría que no existía previamente en nuestra tradición y que se confunde con conceptos como novela o romántico, que, igualmente, resultan un tanto confusos o problemáticos en nuestro idioma. Por contraste, sin salir del ámbito ibérico, la expresión catalana rondalla d'enginy, fácilmente traducible en español como cuento de ingenio, resulta infinitamente más clara y descriptiva y no lleva a ningún tipo de confusión; además, tiene la virtud de calificar estos relatos a partir de uno de sus elementos temáticos esenciales.

2. La traducción al español de la clasificación de Aarne-Thompson realizada por Fernando Peñalosa opta por la traducción absolutamente literal de Novela (Cuentos Románticos). 
Lo terminológico, en cualquier caso, no es sino síntoma de un problema más profundo, justamente el que venimos apuntando desde un principio: la propia definición de la categoría de los cuentos novela. Incluidos estos entre los cuentos folclóricos ordinarios, esto es, entre los cuentos dotados de cierta complejidad estructural y compuestos de varios episodios sucesivos, resulta difícil diferenciarlos de los cuentos maravillosos, si no es a partir de su temática. Así, el propio Thompson alude a lo realista en contraste con lo maravilloso como elemento diferenciador básico entre los subgéneros a los que nos referimos, aunque no oculta que resulta difícil establecer los límites de lo maravilloso y lo realista ante la manera de solucionar en estos relatos ciertos hechos o ante las exageraciones y otros elementos propios de los cuentos novela, que se dirían, desde mi punto de vista, grados mínimos de lo maravilloso (Thompson I972: 209 y ss.). Personalmente, creo que entrar en la diferenciación de lo maravilloso y lo realista dentro del campo de lo ficticio supone meterse en un auténtico problema, poco productivo, por cierto, si no media una categoría que defina con precisión a qué estamos llamando realista. ${ }^{3}$

Lo cierto es que, en términos puramente formales, como señala Rosa Alicia Ramos, solo parecen existir dos grandes categorías dentro de los relatos folclóricos ficticios, que esta autora denomina a partir de los términos alemanes Märchen y Schwanke, y no resulta fácil establecer criterios que no sean los temáticos para distinguir dentro de los Märchen los cuentos maravillosos de los que aquí nos ocupan (Ramos I988).

Frente a esta visión bipolar, Antonio Rodríguez Almodóvar defiende un sistema de triple composición para el cuento folclórico, conformado por los cuentos maravillosos, los llamados por él «de costumbres rurales» y los cuentos de animales (Rodríguez I989: I99). Para este autor, los cuentos de costumbres, entre los que se situarían los cuentos novela, junto con todos los cuentos ordinarios no maravillosos y el amplio campo de los cuentos jocosos, son aquellos

que reflejan los modos de vida de las sociedades agrarias, manteniéndolos o criticándolos; no contienen elementos fantásticos, salvo los que puedan conservar por mimetismo o relación satírica con los cuentos maravillosos. Las principales instituciones reflejadas son la propiedad privada y el matrimonio exógamo. Ellas explican tanto la mayoría de los argumentos como su sentido general, manifiesto o latente (Rodríguez I989: 202).

En esta definición se apuntan ya elementos funcionales que superan la mera diferenciación temática entre los cuentos maravillosos y los cuentos novela, a lo que se añade una perspectiva histórica que el autor desarrolla en las páginas siguientes, señalando que los elementos más característicos de los cuentos de costumbres serían su carácter satírico —-más o menos evidente- y la irrupción del personaje pícaro e ingenioso. Aunque estoy convencido de que los elementos señalados por Rodríguez Almodóvar son los que realmente resultan más productivos para la categorización del subgénero que nos ocupa, su categoría de los cuen-

3. Josep M. Pujol apunta también en esta dirección al hablar de los límites del folclore narrativo (Pujol I995: 67-68). 
tos de costumbres no hace sino generar un nuevo problema para solucionar otro. ${ }^{4}$ Por más que a posteriori sitúe los cuentos novela propiamente en el territorio de transición entre el cuento maravilloso y el cuento de costumbres (Rodríguez I989: 228-229), pasamos de la dificultad de separar los cuentos maravillosos de los cuentos novela a la dificultad de diferenciar estos del amplio conjunto formado por los restantes géneros, entre los que se sitúan ahora los cuentos jocosos, que corresponderían formalmente, junto con los cuentos de animales, a la categoría del Schwanke.

Nuestros añorados Julio Camarena y Maxime Chevalier constataron igualmente este problema al apuntar, en una nota aclaratoria al volumen de su catálogo dedicado a los cuentos novela, la heterogeneidad compositiva, la diversidad de las funciones y, sobre todo, las similitudes entre los argumentos de algunos cuentos novela y de ciertos cuentos jocosos, lo que dificulta con frecuencia su correcta catalogación (Camarena-Chevalier 2003: I3). ${ }^{5}$

Así pues, quizá haya que salir de Aarne-Thompson para buscar la solución de la mano de la menos mala de las clasificaciones genéricas hasta ahora desarrolladas en el folclore; me refiero, claro está, a la etnopoética de Heda Jason. Para ello podemos acudir a la aplicación de la etnopoética al folclore catalán desarrollada por Carme Oriol, quien sitúa los cuentos de ingenio — según la denominación catalana- dentro del cuento no maravilloso, «un tipo de relato que tiene una estructura narrativa compleja [...], pero que no tiene una estructura tipificada como el cuento maravilloso» y que se sitúa dentro del «modo realista, ya que la acción transcurre en un mundo real y no en el mundo de lo maravilloso», aunque, por otra parte, se trata de relatos que «no son susceptibles de ser presentados como ciertos, sino que se ven como una fantasía, como una ficción, y en ello se diferencian de las leyendas» (Oriol 2002: 58). En su definición de los cuentos de ingenio, que tiene la virtud de partir de las categorías, perfectamente establecidas por la etnopoética, de modo, forma, contenido y aspecto social, Carme Oriol, apunta:

En la rondalla d'enginy (AaTh 850-999) s'hi emfatitzen característiques pròpies dels humans com l'enginy, la intel-ligencia, la paciència... En aquest tipus de relat l'heroi o l'heroïna tenen com a antagonistes éssers humans que posseixen atributs inherents a la seva pròpia condició humana. Des del punt de vista formal, algunes rondalles d'enginy contenen un fragment en forma d'endevinalla on es planteja l'enigma que ha de ser resolt (Oriol 2002: 59).

A la luz de esta definición de los cuentos novela como género etnopoético, podría parecer que el problema planteado en un principio ha quedado resuelto de manera definitiva y satisfactoria, por no quedar dudas razonables sobre el hecho de que la categoría que nos ocupa corresponde a un género diferenciable dentro de la clasificación internacional. Sin embargo, aunque confieso que para mí la definición es perfectamente adecuada, planea sobre ella la sombra de una duda

4. Como ocurre con muchas otras ideas de este autor, parece creada simplemente para justificar su proyecto de elaborar una colección de cuentos arquetípicos, que venga a ser una especie de Grimm español.

5. Este mismo problema, el de la posible clasificación de algunos cuentos novela como cuentos jocosos, lo he sufrido también en mi labor de catalogación del cuento folclórico en Aragón; de hecho, es la razón de algunas de las correcciones realizadas en la revisión que llevé a cabo en 1998 (González 1998). 
que plantea de nuevo Luis Beltrán en el artículo al que antes me refería, en el que critica la clasificación creada por Heda Jason. Para Beltrán, quien no resta elogios a las propuestas de la etnopoética, la clasificación resulta coja, precisamente, en la definición del modo etnopoético, en la que no ve sino «la oposición que suele ocupar el lugar de la estética en los estudios literarios de nuestro tiempo: la oposición entre realismo e idealismo» (Beltrán 2002: 73). Si bien Beltrán ve algo de verdad en la propuesta de Jason, entiende que traslada esquemas de la actualidad al mundo de las tradiciones con resultados poco productivos, precisamente y de manera especial en lo relativo al modo realista. Para este autor, no es aceptable la convivencia en un mismo modo de los cuentos novela, la épica y la canción tradicional, cuyos vínculos son a simple vista superficiales (Beltrán 2002: 74). Y aunque no propone un modelo alternativo de clasificación, sí apunta como solución —-desarrollando la clasificación a partir de una teoría estética- el establecimiento de una jerarquización social entre unos géneros elevados, ${ }^{6}$ dirigidos a la educación de la casta gobernante, y unos géneros bajos, dirigidos a todos los niveles, tanto jerárquicos como de edad, y cuya función sería la educación más básica (Beltrán 2002: 75-76). Además, Beltrán propone considerar también en esta teoría estética el carácter mixto serio-cómico de todo género del folclore -independiente del hecho de que ciertos géneros o tipos puedan interpretarse como una carnavalización de otros- y la necesidad de incorporar en la clasificación la estética tradicionalmente agrícola del «idilio tradicional», que caracterizaría a aquellos géneros o textos en que la relación con la tierra natal se expresa mediante la observación del crecimiento familiar (Beltrán 2002: 77-79).

Reuniendo todos estos elementos —que en cierto modo están presentes en la propuesta de Rodríguez Almodóvar sobre los cuentos de costumbres-, cabría pues refinar la definición de Carme Oriol incorporando a las características de los cuentos novela o cuentos de ingenio su carácter de género bajo - por contraste con la épica, aunque no con la canción tradicional-, su naturaleza mixta seriocómica - que en algunos casos se traduce en carnavalización de temas del cuento maravilloso- y la naturaleza idílica de los relatos del conflicto familiar —a los que la propia Heda Jason apunta dentro del modo realista-, entendida esta naturaleza, en palabras de Luis Beltrán, como «una especie de realismo primitivo o tradicional» (Beltrán 2002: 78).

\section{Los cuentos novela en Aragón y La Rioja}

La larga introducción previa se justifica plenamente cuando se aterriza en la realidad, que no se halla en el Catálogo Internacional del Cuento Tipo, sino en las recopilaciones de cuentos realizadas en fechas recientes y en los análisis y catálogos de ámbito más restringido, como los que aquí pretendo presentar, refiriéndome a Aragón y La Rioja. ${ }^{7}$

6. Caracterizados por sus escenarios - el palacio o el templo-, la elevación heroificadora y la expresión de una identidad nacional o de casta, y un tipo especial de palabra, la poética, que suele tener un arropamiento versal y un carácter cerrado, que diríamos irrefutable.

7. Como ya señalé en mi intervención en el encuentro que nos reunió en Pau, en el que hablé de los cuentos jocosos en Aragón y La Rioja, ambas comunidades autónomas españolas comparten suficientes lazos — dada su proximidad y la relación que las vincula a través del valle del Ebro- como para justificar la exposición conjunta de los resultados de la catalo- 
Desde nuestra primera reunión en Toulouse llevo defendiendo este tipo de catalogaciones -incluidos sus excesos a la hora de proponer nuevos tipos- como la piedra de toque en la que debe medirse continuamente la validez y la solidez del Catálogo Internacional, que, de otra manera, podría acabar siendo un corpus muerto y cerrado. En efecto, vamos a ver aquí cómo, paradójicamente, la relativa escasez de tipos y versiones de cuentos novela y el carácter fragmentario de muchos de los recopilados simplifican enormemente la problemática antes señalada y ofrece criterios mucho más productivos para situar los cuentos novela en relación con la sociedad en la que aún perviven.

Antes de ello, me viene a la mente el primer cuento que grabé en mi vida con una grabadora, una versión alavesa —esto es, del ámbito románico vasco- del cuento de los «Tres consejos», o sea, del tipo AaTh 9IoB (González I993: 45-63). Contado por una narradora magnífica, que contextualizaba el relato en un lugar real y que lo titulaba con el nombre de su protagonista, Juan Palomo, el cuento me pareció entonces, con mi pobre bagaje de estudiante, una mezcla de una enorme sátira —en la que se confrontaba la lógica de Juan, prototipo del tonto-listo, con la de su mujer, María, caracterizada por su inteligencia práctica- y un cuento de carácter eminentemente didáctico y sapiencial; esto es, una perfecta simbiosis entre el Märchen, en el que el protagonista consigue alcanzar exitosamente su propósito, y el Schwanke, en el que la torpeza del protagonista parece anunciar continuamente el desastre al que se encamina y, que, sin embargo, en este caso no se consuma. En definitiva, toda una parábola sobre la inteligencia y la sabiduría, que se convierte, en cierto modo, en un buen código de conducta para guiarse de manera práctica en la vida.

Si traigo a colación este relato es porque, como se verá a continuación, los cuentos novela recogidos en Aragón y La Rioja responden igualmente, en su gran mayoría, a esta función claramente educativa y sapiencial; en efecto, en ellos el ingenio y el buen consejo destacan como principios que ofrecen a sus receptores, y convierten el cuento, como queda dicho, en una guía para desenvolverse con éxito y agudeza — no exenta de risa sabia y muy crítica - en el día a día de la sociedad agropecuaria tradicional. Prueba de ello son las versiones fragmentarias, que no debemos justificar solo por un proceso de desgaste o desintegración del relato - fruto en la mayoría de los casos de la pérdida del arte narrativo oral en una sociedad en la que ya no suelen contarse cuentos-, sino, también, por un proceso en virtud del cual los cuentos novela en los que aparecen adivinanzas o respuestas ingeniosas se han ido transformando hasta convertirse en muchas ocasiones en simples interpelaciones del narrador a su auditorio, en las que la adivinanza

\footnotetext{
gación de los cuentos folclóricos recogidos en ambos territorios. Como también señalaba entonces, la parte más exhaustiva de mi trabajo corresponde al territorio aragonés, en el que he realizado varios trabajos de campo y del que vengo desarrollando - con excesivas dificultades de orden práctico y doméstico, dada la falta de apoyos institucionales- un catálogo tipológico (González I996 y I998). Por lo que respecta a La Rioja, mi conocimiento de los cuentos recogidos en esta comunidad se basa en mi colaboración con Javier Asensio, folclorista riojano que ha realizado una magnífica y muy extensa recopilación de cuentos (Asensio 20O2), cuyos resultados analicé y clasifiqué para su publicación. En ambos casos, la recopilación y clasificación se realizó en fechas anteriores a la edición de ATU, por lo que los cuentos, lógicamente, aparecen clasificados conforme a AaTh, aunque también se tienen en cuenta determinadas propuestas de mi propia clasificación y del catálogo de Camarena y Chevalier.
} 
o la respuesta ingeniosa se ofrecen como una medida de la agudeza y del saber popular, y, para el caso que nos ocupa por nuestro oficio, en una verdadera prueba frente al saber enciclopédico del entrevistador o del urbanita. Lo dicho queda especialmente de manifiesto en relación con los tipos AaTh 92ID* y AaTh 929, así como con los distintos subtipos del AaTh 980, quizá los relatos actualmente más vivos en ambos territorios.

Con respecto a los dos primeros, el tipo AaTh 92 $\mathrm{ID}^{*}$, en el que hemos incluido todos los relatos en los que un niño o un campesino tomado por tonto da respuestas ingeniosas y desconcertantes, ha llegado a convertirse en una especie de chiste - como tal suele contarse- en el que los campesinos, como ocurre también con el tipo AaTh 929, ${ }^{8}$ demuestran la superioridad de su ingenio en las agudas respuestas del protagonista - generalmente un niño-, que aparece como una víctima propicia para las burlas de gentes foráneas —en no pocas ocasiones para la guardia civil - y que acaba, sin embargo, burlándose de quienes querían humillarlo - y en no pocas ocasiones, también, humillándolos y hasta insultándolos de forma muy agresiva- ${ }^{9}$

Por lo que respecta a los distintos subtipos de AaTh 980, se diría que sobreviven con especial intensidad en el medio rural de Aragón y La Rioja debido a la despoblación y el envejecimiento de su población rural. De hecho, no son pocos los informantes de avanzada edad que cuentan estos breves relatos apologéticos con gran placer, como si quisieran resarcirse del caso, más que frecuente, del abandono al que se ven sometidos por parte de sus hijos o familiares. ${ }^{\text {Io }}$

Un último asunto, a propósito de la catalogación de los cuentos aragoneses, ha sido la clasificación de ciertos romances, en particular el de «La doncella guerrera» y los que desarrollan el tema del «Amor más fuerte que la muerte» —y contienen el motivo de las ramas enroscadas-, dentro del catálogo de cuentos en razón de sus argumentos, que coinciden con los tipos AaTh 884B* y AaTh 970, respectivamente. Es cierto que no se han hallado cuentos que desarrollen tales temas en prosa, pero con ello se ha querido dejar constancia de la pervivencia de estos argumentos a través de este otro género del folclore narrativo. ${ }^{\text {II }}$

8. La similitud entre los distintos subtipos del tipo AaTh 92I, en particular el AaTh 92ID*, que hemos llegado a convertir en una especie de cajón de sastre, y el tipo AaTh 929, hacen a veces difícil decantarse por uno $\mathrm{u}$ otro ante relatos en los que las respuestas de los campesinos son a un tiempo agudas réplicas y muestras de sabiduría popular.

9. Resulta muy interesante, para el caso de Aragón, la existencia de un género, «la mazada», definido por Rafael Andolz en su obra El humor altoaragonés (Andolz I992: I44 y ss.) como la respuesta rápida e ingeniosa cargada de humor y que desarma de tal manera al interlocutor que no permite réplica. Aunque muchas de las características que Andolz atribuye al humor altoaragonés son en realidad propias del folclore de todos los pueblos, es cierto, y lo defiendo por experiencia propia, que en esta tierra se aprecia de tal manera a la gente ingeniosa - es, al fin y al cabo, la patria de Gracián y de Pedro Saputo- que justificaría la pervivencia en el folclore narrativo de todos los relatos en que la respuesta aguda y rápida o la adivinanza o prueba que debe ser resuelta con ingenio son constituyentes esenciales de su trama.

Io. Habría que añadir aquí que, en los diversos trabajos de campo que he realizado, he acudido con frecuencia a residencias de ancianos y hogares de jubilados, donde me he encontrado con tragedias personales verdaderamente lamentables. En este contexto, los relatos del hijo ingrato que es reprobado por las acciones ingenuas de su propio hijo cobran un sentido especial.

II. Sería interesante reflexionar sobre las relaciones entre el romance y los cuentos novela a la luz de estos y de otros posibles ejemplos. Es posible que de este modo estuviéramos, 
Basten, en fin, estas líneas previas para valorar los resultados de las recopilaciones y catalogaciones realizadas en Aragón y La Rioja, que, dado que no hay espacio para mucho más, expongo a continuación en términos meramente cuantitativos.

\subsection{Los cuentos novela en Aragón}

A partir de la última versión de mi catálogo (González I998), los tipos y versiones registrados en Aragón, separados por dominios lingüísticos, son los siguientes:

DOMINIO LINGÜÍSTICO ARAGONÉS

1 versión oral del tipo 889 Wager on the Faithfulness of the Servant.

\section{DOMINIO LINGÜÍSTICO CASTELLANO}

1 versión oral del tipo 85I The Princess who Cannot Solve the Riddle.

1 versión oral del tipo 860 Nuts of «Ay, ay, ay».

1 versión literaria del tipo AaTh 875 The Clever Peasant Girl.

2 versiones literarias del tipo 879 The Basil Maiden. ${ }^{\mathrm{I2}}$

5 versiones orales del tipo AaTh 884 B $^{*}$ Girl Dressed as Man is Deceives the King (ATU

884) —-todas del romance «La doncella guerrera»-.

1 versión oral del tipo 9or Taming of the Shrew.

1 versión oral del tipo 9Io The Clever Precepts.

1 versión oral y 2 literarias del tipo 9IоB The Observance of the Master's Precepts.

3 versiones orales y una literaria del tipo 92 $\mathrm{ID}^{*}$ Witty Answers.

2 versiones orales del tipo AaTh 922 The Shepherd Replacing the Priest Answers the King's Questions.

2 versiones orales y una literaria del tipo 923 Love Like Salt.

1 versión literaria del tipo 952 The King and the Soldier.

1 versión oral del tipo 954 The Forty Thieves.

3 versiones orales del tipo 958 The Shepherd Youth in the Robber's Power.

1 variante oral del tipo 960A The Cranes of Ibycus.

1 versión oral del tipo 967 - se trata de una leyenda religiosa- The Man Saved by a Spider Web.

10 versiones orales del tipo 970 —-todas son romances del «Conde niño» que desarrollan el tema del «Amor más poderoso que la muerte»- The Twining Branches.

precisamente, entrando en la cuestión antes apuntada de la relación existente entre relatos ficticios de carácter realista, diferenciados en este caso no solo por su forma - verso/prosa-, sino también por su categoría de género alto o bajo.

I2. Todas las versiones catalogadas hasta la fecha contienen únicamente los episodios I, II y III. Solo recientemente, en una recopilación en curso en la comarca aragonesa de Valdejalón, dirigida por Carolina Ibor, se ha recogido una versión completa del tipo que incluye el episodio IV The sugar puppet. 
2 versiones orales y una literaria del tipo AaTh 98oB Wooden Drinking Cup for Old Man.

DOMINIO LINGÜÍSTICO CATALÁN

1 versión oral del tipo 853 The Hero Catches the Princess with her Own Words.

1 versión oral del tipo 877 The Old Woman Who Was Skinned.

2 versiones orales del tipo 879 The Basil Maiden.

1 versión oral del tipo 900 King Thrushbeard.

1 versión oral del tipo 9or Taming of the Shrew.

2 versiones orales del tipo AaTh 920A The Daughter of the King and the Son of the Peasant.

1 versión oral del tipo 923 Love Like Salt.

3 versiones orales del tipo 929 Clever Defenses.

1 versión oral del tipo 950 Rhampsinitus.

4 versiones orales del tipo AaTh 980B Wooden Drinking Cup for Old Man.

10 versiones orales del tipo AaTh 980C Dragging Old Man only to Threshold.

\subsection{Los cuentos novela en La Rioja}

A partir de los resultados obtenidos en la recopilación de Javier Asensio (Asensio 2002), los tipos y versiones registradas en esta comunidad —orales en su totalidad-son los siguientes:

1 versión del tipo 85I The Princess who Cannot Solve the Riddle.

1 versión del tipo 860 Nuts of «Ay, ay, ay».

3 versiones del tipo 9or Taming of the Shrew.

9 versiones del tipo 921D* Witty Answers.

1 versión del tipo 923 Love Like Salt.

1 versión del tipo 929 Clever Defenses.

1 versión del tipo 933 Gregory on the Stone.

1 versión del tipo 95IA The King and the Robber.

2 versiones del tipo 958 The Shepherd Youth in the Robber's Power.

1 versión del tipo AaTh 964 Thief (Murderer) Deceived into Betraying himself by a Gesture.

1 versión del tipo AaTh 980A The Half-Carpet - Javier Asensio señala al respecto de este tipo y de los subtipos AaTh 980B y AaTh 980 C que son «universalmente conocidos», por lo que solo recoge, a modo de ejemplo, las tres versiones narradas por dos hermanos informantes-.

1 versión del tipo AaTh 980 B Wooden Drinking Cup for Old Man.

1 versión del tipo AaTh 980 C Dragging Old Man only to Threshold.

1 versión del tipo 982 The Pretended Inheritance. 


\subsubsection{Versiones no catalogadas de posibles cuentos-novela}

«El tío Pedro León» (Asensio 2002: I47-I49): el tío Pedro León busca sus yeguas, que se han escapado, en la casa de unos gitanos. Huye de la casa al oír a los hijos de los gitanos que quieren comer sus «pezuñitas». Cuando escapa, los gitanos azuzan a sus perros, animándolos con la promesa de repartir entre ellos las distintas partes del cuerpo del tío Pedro. En su momento propusimos su catalogación como una variante, por su semejanza, de AaTh 958B*, aunque señalábamos también sus semejanzas con AaTh II49.

«La princesa y el quincallero" (Asensio 2002: I34-I37): los padres de un niño que solo sabe decir «zambombo» ${ }^{13}$ deciden dejárselo a un quincallero - por su fama de charlatanes - para que aprenda a hablar, dado que debe ser capaz de recitar un poema para poder casarse. El hijo vende a una princesa un anillo, unos pendientes y una pulsera, cambiándoselos por un beso, un abrazo y el vestido que lleva puesto. Hace creer al quincallero que solo sabe decir «zambombo» y este lo devuelve a los padres. Se casa con la princesa y sorprende a estos, que creían que no sabía hablar, con un relato en el que, simbólicamente, narra sus encuentros previos con la princesa. Es semejante a Boggs $900^{*} \mathrm{~A}$ y Espinosa n. ${ }^{\circ} 234$.

«El abuelo que cambió el testamento» (Asensio 2002: I5I): el abuelo cambia el testamento al ver que, tras salir del notario, no le permiten volver a su pueblo montado en el burro, en el que el hijo — que lo ha heredado todo- ha montado al nieto.

I3. Según la Real Academia Española, se trata de una palabra de uso coloquial que significa «Hombre tosco, grosero y rudo de ingenio». No obstante, se diría que en el cuento se usa como palabra sin sentido aparente, casi como mera interjección. 


\section{Referencias bibliográficas}

ANDOLZ, Rafael (1992): El humor altoaragonés. Zaragoza: Mira.

ASENSIO, Javier (2002): Cuentos riojanos de tradición oral. Logroño: Gobierno de La Rioja.

BELTRÁN AlmeríA, Luis (2002): «Géneros y estéticas en la literatura tradicional». Revista de Literaturas Populares, núm. 2: 67-8I.

CAMARENA, Julio; Maxime CHevalier (2003): Catálogo tipológico del cuento folklórico español. Cuentos-novela. Alcalá de Henares: Centro de Estudios Cervantinos.

EsPinosA, Aurelio M. (I996-97): Cuentos populares de Castilla y León. Madrid: Consejo Superior de Investigaciones Científicas.

GonzÁlez SAnz, Carlos (ed.) (I993): Cuentos populares de Kanpezu. Recogidos de boca de Macaria Iriarte. Vitoria: Diputación Foral de Álava.

- (I996): Catálogo tipológico de cuentos folklóricos aragoneses. Zaragoza: Instituto Aragonés de Antropología.

- (I998): «Revisión del Catálogo tipológico de cuentos folklóricos aragoneses: correcciones y ampliación». Temas de Antropología Aragonesa, núm. 8: 7-6o.

OrIOL, Carme (2002): Introducció a l'etnopoètica. Teoria i formes del folklore en la cultura catalana. Valls: Cossetània Edicions.

Pujol, Josep. M. (I995): «Sobre els límits del folklore narratiu». Estudis Baleàrics, núm. 52: 63-74.

RAMos, Rosa Alicia (I988): El cuento folklórico: una aproximación a su estudio. Madrid: Pliegos.

RODRÍGUEz ALMODÓvAR, Antonio (I989): Los cuentos populares o la tentativa de un texto infinito. Murcia: Universidad de Murcia.

Thompson, Stith (I972): El cuento folklórico. Caracas: Universidad Central de Venezuela.

UTHER, Hans-Jörg (2005): «La nouvelle classification internationale des contestypes (ATU)». Cahiers de Littérature Orale, núm. 57-58: 225-237. 